\title{
Alternative Splicing in Acad8 Resulting a Mitochondrial Defect and Progressive Hepatic Steatosis in Mice
}

\author{
NAGHAM GEORGE ABD AL-AHAD SABBAGHA, HSIAO-JUNG KAO, CHIH-FU YANG, CHENG-CHIH HUANG, WEI-DE LIN, \\ FUU-JEN TSAI, TZU-HO CHEN, WOAN-YUH TARN, JER-YUARN WU, AND YUAN-TSONG CHEN \\ Institute of Biomedical Sciences [N.G.A.S., H.-J.K., C.-F.Y., C.-C.H., T.-H.C., W.-Y.T., J.-Y.W., Y.-T.C.], Academia Sinica, Taipei 11529, \\ Taiwan; Institute of Biochemistry and Molecular Biology [N.G.A.S.], National Yang-Ming University, Taipei 11221, Taiwan; Department \\ of Medical Research [W.-D.L., F.-J.T., J.-Y.W.], China Medical University Hospital, Taichung 40408, Taiwan
}

\begin{abstract}
Using a combination of $N$-ethyl- $N$-nitrosoureamediated mutagenesis and metabolomics-guided screening, we identified mice with elevated blood levels of short-chain C4-acylcarnitine and increased urine isobutyryl-glycine. Genome-wide homozygosity screening, followed by fine mapping, located the disease gene to $15-25 \mathrm{Mb}$ of mouse chromosome 9 where a candidate gene, Acad8, encoding mitochondrial isobutyryl-CoA dehydrogenase was located. Genomic DNA sequencing revealed a single-nucleotide mutation at -17 of the first intron of Acad 8 in affected mice. cDNA sequencing revealed an intronic 28 -bp insertion at the site of the mutation, which caused a frame shift with a premature stop codon. In vitro splicing assay confirmed that the mutation was sufficient to activate an upstream, aberrant $3^{\prime}$ splice site. There was a reduction in the expression of Acad8 at both the mRNA and protein levels. The mutant mice grew normally but demonstrated cold intolerance at young age with a progressive hepatic steatosis. Homozygous mutant mice hepatocytes had abnormal mitochondria with crystalline inclusions, suggestive of mitochondriopathy. This mouse model of isobutyryl-CoA dehydrogenase deficiency could provide us a better understanding of the possible role of IBD deficiency in mitochondriopathy and fatty liver. (Pediatr Res 70: 31-36, 2011)
\end{abstract}

$\mathrm{T}$ The branched-chain amino acids valine, isoleucine, and leucine are structurally similar. Valine and isoleucine can both be converted to glucose (1). Nitrogen is removed from valine in peripheral tissues (e.g. muscles) by the branched-chain amino acid transaminase. During exercise or fasting, $\alpha$-ketoisovalerate is released into the circulation and transported to the gluconeogenic organs, the liver and kidneys (2), where carnitine acyltransferases are responsible for the maintenance of CoA levels (3), and $\alpha$-ketoisovalerate is irreversibly converted to isobutyryl-CoA, where it is oxidized by isobutyryl-CoA dehydrogenase (IBD), encoded by Acad8, toward the gluconeogenic pathway (4). IBD is a member of a dehydrogenase family of mitochondrial enzymes that are synthesized in the cytosol and imported into the mitochondrial matrix with an activesite glutamate located in its C-terminus (5).

Correspondence: Yuan-Tsong Chen, M.D., Ph.D., Institute of Biomedical Sciences, Academia Sinica, No.128, Sec.2, Yen-Jiou-Yuan Rd., Taipei 11529, Taiwan; e-mail: chen0010@ibms.sinica.edu.tw

Supported by Academia Sinica Genomics and Proteomics Program; and Taiwan Mouse clinic Core NSC97-3112-B-001-025.

Supplemental digital content is available for this article. Direct URL citations appear in the printed text and are provided in the HTML and PDF versions of this article on the journal's Web site (www.pedresearch.org).
The recent development of tandem mass spectrometry makes it possible to screen newborns for more than 20 inborn errors of metabolism including IBD deficiency. The elevated levels of C4-acylcarnitine in blood spots samples, together with the detection of isobutyryl-glycine in the urine, rendered it possible to differentiate IBD deficiency from a short-chain acyl-CoA dehydrogenase deficiency(SCAD) which also results in increased levels of C4-acylcarnitine. The first documented patient with IBD deficiency was described by Roe $e t$ al. (6). Additional patients were subsequently reported by other groups (7-10). These patients ranged from being asymptomatic, to have muscular hypotonia, mild developmental delay, speech delay, and one with reversable cardiomyopathy. Oglesbee et al. (11) had developed a newborn screening follow-up algorithm for the diagnosis of the disease.

Animal models play an important role in the study of the pathophysiology of human metabolic diseases by providing the opportunity to discover the underlying mechanism of a particular disorder (12). $N$-ethyl- $N$-nitrosourea (ENU) is a chemical mutagen that when injected into mice can efficiently generate single-nucleotide mutations in the mouse genome. Mice carrying the mutations can then be screened for disease phenotypes of interest. By systematic metabolic screening, we previously reported mice with clinical features resembling human maple syrup urine disease (13). We have also identified a mouse model of human long-chain 3-hydroxyacyl-CoA dehydrogenase deficiency (14). Here, using a similar approach, we report mice with IBD deficiency due to a mutation in Acad8.

\section{MATERIALS AND METHODS}

Generation of mice with recessive mutations. Breeding and housing of the mice were conducted in the Mouse Mutagenesis Program Core Facility of Academia Sinica under specific pathogen-free conditions. The Academia Sinica Institutional Animal Safety Committee approved the animal protocol. ENU-treated mice were bred according to a three-generation breeding scheme as previously described (15).

Metabolic screening and biochemical analysis. Quantitative plasma tandem mass spectrometry (MS/MS) was performed on whole blood sample spotted on a Guthrie card for metabolic screening of amino acids and

Abbreviations: BAT, brown adipose tissue; ENU, $N$-ethyl- $N$-nitrosourea; IBD, isobutyryl-CoA dehydrogenase; RER, respiratory exchange rate; SGOT, serum glutamic oxaloacetic transaminase; SGPT, serum glutamic pyruvate transaminase; SNP, single-nucleotide polymorphism 
acylcarnitine species. Screening of urinary organic acids was performed after collection of $100 \mu \mathrm{L}$ of urine from each mouse using capillary column gas chromatography as previously described (13). Plasma samples were analyzed using a FUJI DRI-CHEM 3500s (Fuji Photo Film, Tokyo, Japan) for measurement of glucose, serum glutamic oxaloacetic transaminase (SGOT), serum glutamic pyruvate transaminase (SGPT), creatinine, triglycerides, and cholesterol.

Mapping and identification of the mutant gene. A total genome-wide mouse 262 single-nucleotide polymorphisms (SNPs) were selected for genotyping based on the criterion that the genotypes of the mouse strain $\mathrm{C} 3 \mathrm{H} / \mathrm{HeJ}$, $\mathrm{DBA} / 2 \mathrm{~J}$, or $\mathrm{BALB} / \mathrm{cByJ}$ are different from that of C57BL6J. The average space between each SNP was $10 \mathrm{Mb}$. SNP genotyping using genomic DNA isolated from mouse tails was performed by high-throughput MALDI-TOF mass spectrometry (14). Primers and probes flanking the SNPs were designed in a multiplex format using SpectroDESIGNER software (Sequenom, San Diego, CA).

DNA sequencing. Genomic DNA was purified using a Puregene DNA Purification kit (Gentra Systems, Minneapolis, MN). All exons, exon-intron junctions, and $2.5 \mathrm{~kb}$ of the promoter region of the candidate gene, Acad8, and Scad were amplified and sequenced. Primers were designed using Primer3 software (http://www.genome.wi.mit.edu/cgi-bin/primer/ primer3_www.cgi). The primers used for the detection of intron- 1 mutations in the Acad8 gene were 5'-GATAGCAGAGGCAGGATGGT-3' (sense strand, in intron 1) and 5'-CCTTCTACCCACCAACTCCA-3' (antisense strand, in intron 2). PCR was performed as previously described (14).

cDNA sequencing and quantitative real-time QPCR. For cDNA sequencing, and to examine the differences in the tissue expression of Acad 8 and genes of the $\beta$-oxidation and de novo lipogenesis pathways, total RNA samples were extracted from the liver and kidney of 2-mo-old mutant and WT in both fasting and nonfasting status using Trizol (Invitrogen, Carlsbad, CA) following the manufacture's protocol. QPCR were done as described before (Table S1, http://links.lww.com/PDR/A71) (13).

In vivo splicing assay. The Acad8 minigene contained the entire exons 1 and 2 and an internally truncated intron 1 which comprised 400 nucleotides at the $5^{\prime}$ and $3^{\prime}$ ends. This minigene was constructed in the pCH110 plasmid (Amersham Pharmacia, Piscataway, NJ), and its expression was under the control of the SV40 promoter. The minigene was transiently transfected into mouse myoblast $\mathrm{C} 2 \mathrm{C} 12$ cells using Lipofectamine (Invitrogen, Carlsbad, CA). Twenty hours posttransfection, total RNA was extracted from the transfectants and subjected to RT-PCR using an SV40 forward primer and a reverse primer complementary to Acad8 exon 2 (5'-ATCGGGTACCCTTCTGATCCCACTCCGCCA- $3^{\prime}$ ). The PCR products were electrophoresed on a urea containing $8 \%$ polyacrylamide gel and detected by Southern blot using a 32P-labeled SV40 probe (16).

Western blot analysis. Mitochondrial fractions were isolated from mouse livers and kidneys using a mitochondria isolation kit (MITO-ISO1; Sigma Chemical Co. Aldrich, St. Louis, MO). Whole cell lysates of brown adipose tissue (BAT) from both WT and homozygous mutant mice were obtained using RIPA buffer. Western blot analysis was performed after the separation of $50 \mu \mathrm{g}$ of protein via $10 \%$ SDS-PAGE. Goat polyclonal antibodies recognizing the $N$-terminus of the mouse Acad8 protein (1:200; Santa Cruz Biotechnology), goat anti-human heat shock protein (Hsp60) polyclonal antibody (Stressgen Biotechnologies Corporation, San Diego, CA) as an internal control, rabbit polyclonal antibodies against mitochondrial uncoupling protein 1 (UPC1; Abcam, Cambridge, MA) at a dilution of 1:1000, and anti- $\beta$-actin antibody as an internal control were used.

Metabolic rate measurement. All measurements were performed in a PhenoMaster System (TSE Lab Master System, Homburg, Germany), which allows measurement of metabolic performance. Four WT and four mutant mice were placed at room temperature $\left(22^{\circ} \mathrm{C}-24^{\circ} \mathrm{C}\right)$ in the chambers of the PhenoMaster open circuit calorimetry. Mice were allowed to adapt to the chambers for at least $24 \mathrm{~h}$. Food and water were provided ad libitum in the appropriate devices and measured by the build-in automated instruments. Parameters of indirect calorimetry, $\mathrm{VO}_{2}$ and $\mathrm{VCO}_{2}$ production, respiratory exchange rate (RER), and food intake were measured for the following $48 \mathrm{~h}$. The capacity of the WT and mutant mice to increase their metabolic rate was determined by measuring their $\mathrm{VO}_{2}$ before and after an injection of 1000 $\mathrm{mg} / \mathrm{kg}$ of body weight L-noradrenaline as described in Ref. 17. Furthermore, animals were put in running wheels equipped with a speedometer (Sigma Chemical Co. Sport BC700 calibrated for running wheel radius) fitted to the individual plastic cage with grill lid. The distance run $(\mathrm{km})$ and speed $(\mathrm{km} / \mathrm{h})$ were measured. Micro CT was performed to measure body fat volume for three WT and three mutant mice (Sky Scan, Belgium).

Cold intolerance test. At the age of $4 \mathrm{wk}$, six male WT and six homozygous mutant mice were subjected to cold intolerance tests after fasting overnight. After measuring their basal rectal body temperature under thermoneutral conditions, the mice were put at $4^{\circ} \mathrm{C}$ with free access to water and food. Rectal body temperature was monitored once hourly for $6 \mathrm{~h}$ using a TES-1306 thermometer probe introduced $1-2 \mathrm{~cm}$ in the rectum.

Histopathology. Three affected male mice of 4-, 12-, and 40-wk-old and age-matched control mice were killed by i.p. injection of $66 \mathrm{mg} / \mathrm{kg}$ sodium pentobarbital then perfused through the heart with $4 \%$ paraformaldehyde in 0.1 M PBS (pH 7.4). Liver, heart, kidney, stomach, intestine, spleen, brain, retina, skin, white adipose tissue and BAT, and thigh muscles were then removed, processed, and stained with hematoxylin-eosin (14)

Transmission electron microscopy. Three animals of each age group including $(4,12,30$, and $40 \mathrm{wk})$ of both WT and mutant mice were killed with i.p. injection of pentobarbital $(66 \mathrm{mg} / \mathrm{kg})$. Intracardiac perfusion with $2.5 \%$ glutaraldehyde in $0.1 \mathrm{M}$ phosphate buffer was performed, and liver biopsies were processed as previously described (18). Individual hepatocytes were examined for evaluation of the mitochondria.

\section{RESULTS}

Mutant mice with abnormal metabolites. In a recessive screen of $2300 \mathrm{G} 3$ mice from 61 families at 2-3 mo of age; we identified mice with elevated blood levels of $\mathrm{C} 4$ acylcarnitines $(1.549 \pm 0.163 \mu \mathrm{M})$ compared with WT mice $(0.417 \pm 0.169$ $\mu \mathrm{M}$; Fig. $1 A$ ). Furthermore, they showed a significant reduction in blood-free carnitine $(21.96 \pm 1.726 \mu \mathrm{M})$ compared with WT mice (35.97 $\pm 8.036 \mu \mathrm{M}$; Fig. 1B). All other short-,
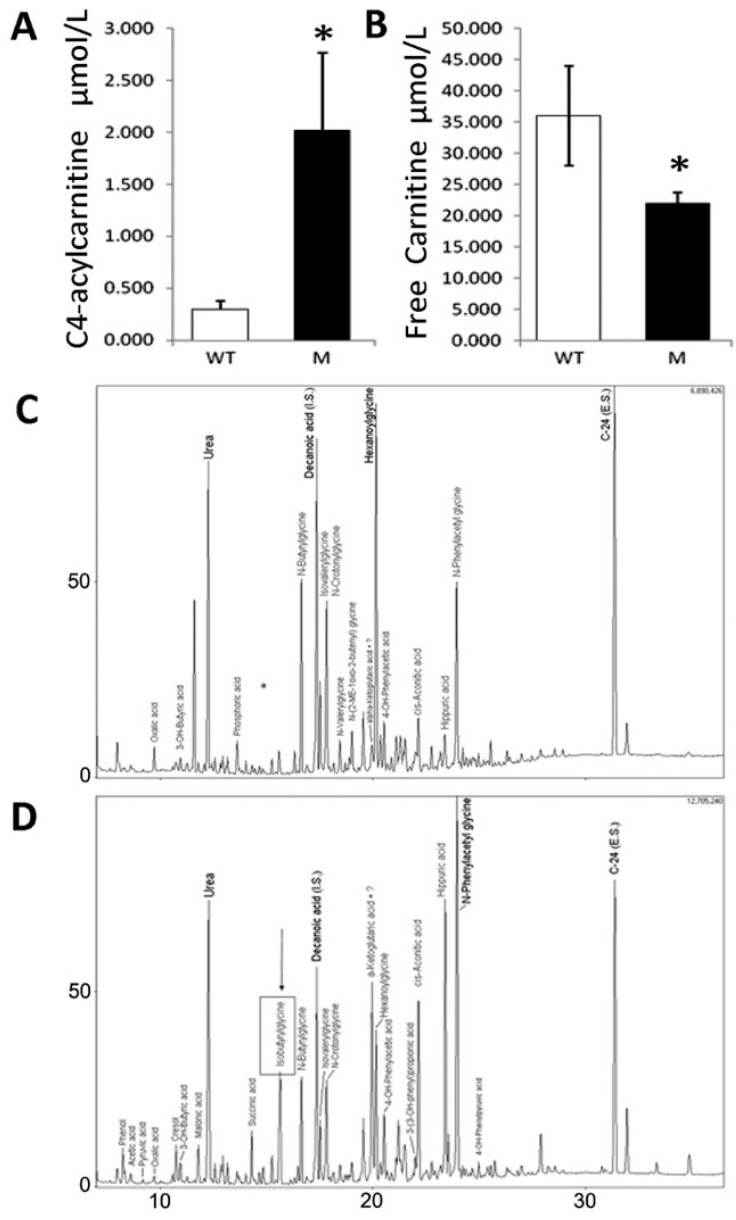

Figure 1. Metabolic screening. (A) Blood C4-acylcarnitine concentrations in mutant mice, M (black bar) compared with WT (white bar); each bar represents mean of six mice \pm SD for each group. $(B)$ Blood-free acylcarnitine concentrations in mutant mice (black bar) compared with WT (white bar); each bar represents mean of six mice $\pm \mathrm{SD}$ for each group. $(C, D)$ Urine organic acid profiles show the appearance of isobutyryl-glycine in the urine samples of mutant mice (arrow) compared with the WT trace (star). $* p<0.01$. 
medium-, and long-chain acylcarnitines and blood amino acid levels were normal. The presence of elevated C4-acylcarnitines was confirmed in a second sample from the same mice obtained 4 wk later. These G3-affected mice were then mated with C57BL/6 (B6) to generate F1, and F2 offspring were generated from $\mathrm{F} 1$ brother and sister crosses. Of the $85 \mathrm{~F} 2$ mice, $20(23.5 \%)$ showed a similar elevation in C4acylcarnitine levels, and all F1 mice were normal, indicating that this biochemical abnormality is heritable and transmitted as an autosomal recessive. Urine analysis revealed the presence of isobutyryl-glycine in affected mice compared with the trace amount measured in unaffected mice (Fig. $1 C$ and $D$ ).

Identification of the Acad8 mutation. Homozygosity mapping with high-density SNPs located the mutant gene to chromosome 9 (Fig. S1A, http://links.lww.com/PDR/A71). Fine mapping of $15 \mathrm{~N} 1 \mathrm{~F} 1$ mice further narrowed the region with complete homozygosity between the consecutive SNPs at positions 15736107 and 28250173 of chromosome 9. Within this region, the Acad 8 gene was the most likely candidate. There were no other known genes related to steatosis, fatty acid oxidation, or mitochondrial dysfunction. Sequencing of Acad8 revealed a point mutation 17 bases upstream of exon 2 (IVS1-17T $>$ C; Fig. 2A). Further study of siblings showed that this mutation faithfully segregated with the elevated $\mathrm{C} 4$ shortchain acylcarnitine phenotype. Siblings and parents who were heterozygous for this mutation exhibited normal acylcarnitine profiles. No other mutations were found in the exons of Acad8 or in its promoter region.

Sequencing of the Acad 8 cDNA revealed a 28 bp insertion in intron 1 (r.103_104ins28) (Fig. 2B). Alignment of nucleotide and deduced amino acid sequences derived from the cDNA revealed a frame-shift mutation with a premature stop codon that predicted the production of a truncated protein ending at a lysine residue at position 50 (p.Asp34fsX16) (Fig $\mathrm{S} 1 B$, http://links.lww.com/PDR/A71).

Acad8 expression analysis. QPCR showed that the expression of the Acad8 mRNA was significantly reduced in the tissues of affected mice, including the liver and kidneys, compared with WT controls (Fig. 2C). Western blot analysis in the liver and kidney showed that all the homozygous mutant mice were lacking Acad8 expression when compared with WT (Fig. 2D). This observation was consistent with a frameshift mutation and premature stop codon, which leads to diminished expression of Acad8 protein.

In vivo splicing assay. Because the identified mutation was not at the splice junction, we performed an in vivo splicing assay to determine whether the point mutation was sufficient to activate an upstream aberrant $3^{\prime}$ splice site. The Acad 8 minigens (Fig. 3A) were transiently transfected into mouse myoblasts. RT-PCR analysis showed that the WT minigene produced both the authentic and aberrantly spliced RNAs, of which the former was dominant (Fig. 3B, lane 2); however, the mutation in the minigene greatly increased aberrant splicing (Fig. 3B, lane 3). The same result was also observed in human embryonic kidney 293 cells (data not shown). Therefore, the Acad 8 point mutation was sufficient to activate the use of the upstream aberrant $3^{\prime}$ splice site. We suspected that the resulting aberrant transcript may acquire a premature stop
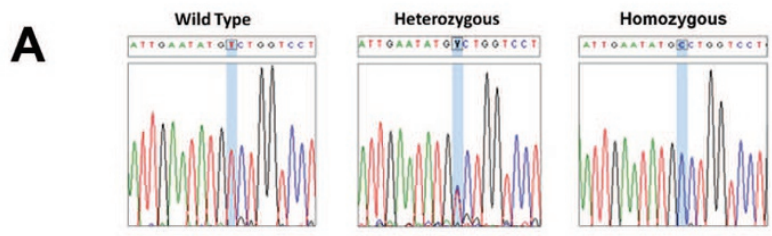

B
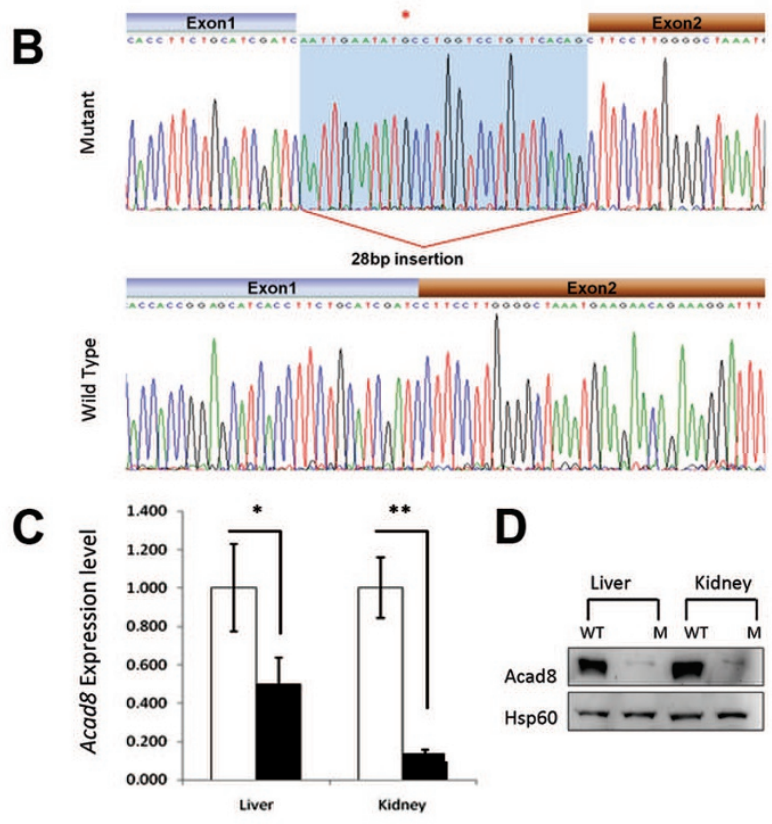

Figure 2. Molecular analysis of the gene responsible for the phenotype. $(A)$ Mouse Acad8 genotyping. Nucleotide sequences of intron 1 shows the WT, heterozygous, and homozygous mutant at position IVS1-17T $>\mathrm{C}$ (blue bar). $(B)$ Sequencing of the cDNA from mutant mice shows the mutation site in intron 1 (star) and the 28 bp insertion between exons 1 and 2. $(C)$ Relative expression level of Acad8 in liver and kidney of WT, white bars and mutant, black bars (each bar represents mean of four mice \pm SD for each group). This reaction was performed in triplicate and analyzed as in Fig. S2. ${ }^{*} p<0.05$, $* * p<0.001$. (D) Western blots analysis in the liver and kidney shows the absence of Acad8 expression in mutant mice.

codon in exon 2 and is thus subjected to nonsense-mediated mRNA degradation. Therefore, the expression of Acad8 protein was reduced.

Peroxisome proliferator-activated receptors (PPAR) and liver $X$ receptor $(L X R)$ pathways expression analysis. At the basal levels, Ppar- $\alpha$ and Ppar- $\gamma$ showed a significant lower level in mutant compared with the WT. Cpt1 and Cpt2, however, showed no significant difference at basal levels. Cd36 showed a significant increase in the mutant after fasting. In contrary, genes involved in the LXR pathway, Lxr-a/b, AccI, Acl, Srebp1c, Scd1, and Fas showed significantly higher expression in the mutants at the basal levels (Fig. S2, http://links.lww.com/PDR/A71).

Clinical phenotypes. Apart from their abnormal metabolic screening results, the affected mice developed normally to adulthood. There was no difference in mutant life span, fertility, or weight gain from the WT (data not shown). There was no fasting hypoglycemia (115.7 $\pm 34 \mathrm{mg} / \mathrm{dL}$; range, 91-189) in mutant mice when compared with the fasting glucose levels of the WT $(122.3 \pm 28 \mathrm{mg} / \mathrm{dL}$; range, 95-180; $p=0.613$ ). Moreover, glucose tolerance test was normal (data 


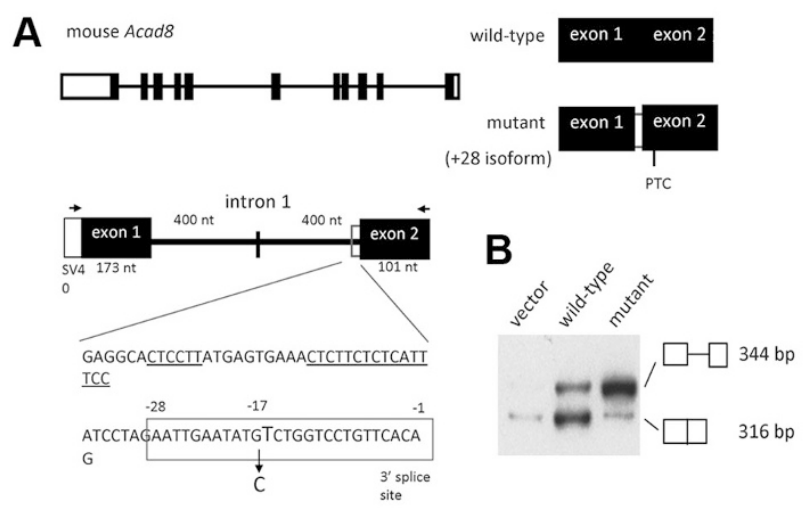

Figure 3. The intronic mutation in Acad8 activates the use of an aberrant $3^{\prime}$ splice site. (A) The upper diagram shows the mouse Acad8 primary transcript and the WT and mutant isoforms. PTC represents premature stop codon. The lower diagram shows the Acad8 minigene containing exons 1 and 2 and internally truncated intron 1 . The mutant contained a $\mathrm{T}$ to $\mathrm{C}$ mutation at the -17 position of intron 1 . Boxed intronic sequence is included in the aberrantly spliced +28 isoform. Underlined sequences represent putative polypyrimidine tract. Arrows indicate a set of primers used for RT-PCR. $(B)$ The WT and mutant Acad 8 minigenes were transiently transfected into mouse $\mathrm{C} 2 \mathrm{C} 12$ cells. Total RNA s were analyzed by RT-PCR followed by Southern blotting. Lane 1 shows mock transfection using the empty vector.

not shown). Both groups of mice demonstrated normal liver and kidney function based on levels of SGOT, SGPT, triglyceride, cholesterol, and blood urea. However, at the age of $40 \mathrm{wk}$, the mutant mice showed significantly elevated transaminase levels (SGOT: $296.6 \pm 81.9$; SGPT: $18.16 \pm 20.4$ ) compared with WT (SGOT: $53.17 \pm 4.7$; SGPT: $44.16 \pm 8.7 ; p<0.005$ ).

Metabolic rate and cold intolerance. During the second day of measurement, mutant mice showed an alteration in the normal diurnal variation of rodent energy expenditure (Fig. 4A). The overall RER and other parameters such as food intake, body activity, and body fat were not significantly different in both groups (Fig. S3, http://links.lww.com/PDR/A71).

The s.c. injection of noradrenaline significantly increased the $\mathrm{VO}_{2}$ above the resting stage level in WT but not the mutant (Fig. $4 B$ ). Examination of mutant mice immediately after weaning revealed that they were less tolerant to cold exposure, demonstrating a significant drop in their rectal body temperature compared with WT control mice, suggesting a defect in the adaptive thermogenesis (Fig. 4C). However, Pgcla expression level in BAT showed significant increase after cold exposure with no significant difference between WT and mutant (Fig. 4D). BAT Ucp1 was detected equally (via Western blot) in both WT and mutant mice after cold exposure (Fig. 4E). Examination of 12- and 20-wk-old mutant mice showed that they were tolerant to cold with the ability to maintain normal rectal body temperature.

Histopathological examination. Compared with WT (Fig. $5 A$ ), mutant mice at $4 \mathrm{wk}$ of age showed early signs of microvesicular steatosis in the form of ballooning of hepatocytes with clarification of the cytoplasm and multiple small fatty inclusion bodies compared with WT (Fig. 5B) and cytoplasmic red bodies under higher magnifications (Fig. 5C). Twelve-week liver showed the progression of microvesicular to macrovesicular steatosis with mononuclear cells infiltration
A
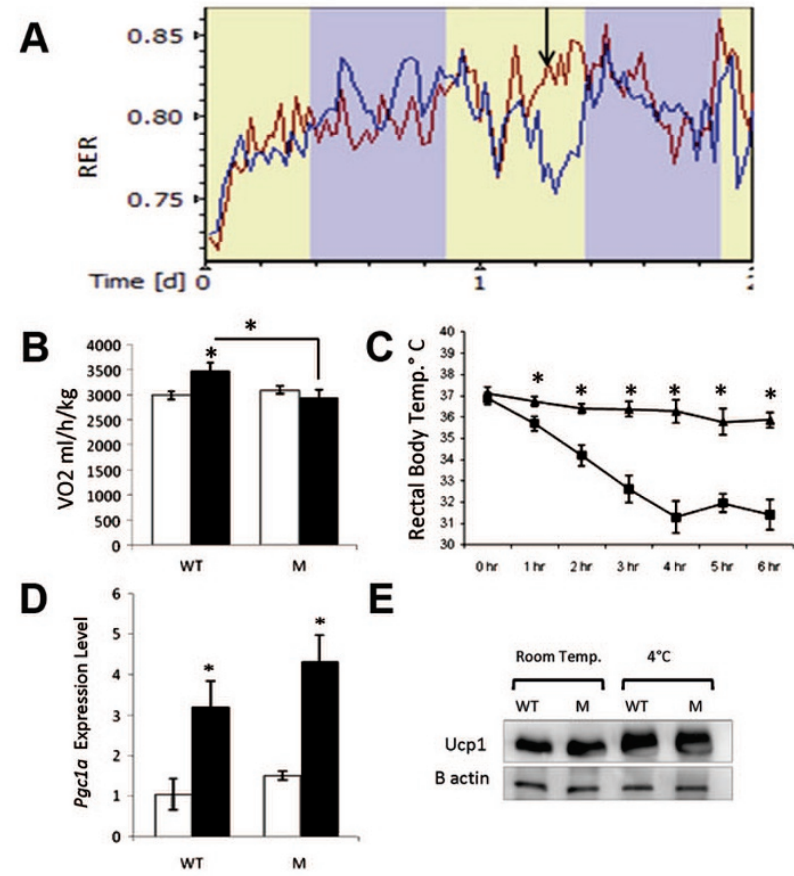

Figure 4. Metabolic rate and cold intolerance test. (A) $48 \mathrm{~h}$ record of the RER of WT (blue line) and mutant (red line) shows that the mutant mice lost their diurnal alteration during the second day. Blue color represents dark cycle and the yellow color represents the light cycle ( $n=4$ animals per group). $(B)$ Noradrenalin induced elevation of the $\mathrm{VO}_{2}$ in WT but not the mutant mice, white bars represent resting stage, and black bars represents $\mathrm{VO}_{2}$ after noradrenalin (each bar represents mean of four mice \pm SD for each group). ${ }^{*} p<0.01$. (C) Differences in rectal body temperature between 4-wk-old WT $(\boldsymbol{\Delta})$ and mutant mice $(\square)$ during 6-h cold exposure each point represent mean of five animals $\pm \mathrm{SD} * p<0.001$. (D) Pgclexpression level in BAT (white bars) shows significant increase after cold exposure (black bars) in both WT and mutant with no difference between the two groups after cold exposure (each bar represents mean of four mice $\pm \mathrm{SD}$ for each group). ${ }^{*} p<0.05$. (E) Western blot analysis of WT and mutant mice BAT before and after exposure to cold shows no significant difference in Ucp1 expression.

(Fig. 5D). Finally, examination of 40-wk-old mutant mice showed macrovesicular steatosis (Fig. $5 E$ ) with foci of mononuclear cell infiltration (Fig. $5 F$ ). Sections of BAT from mutant mice revealed smaller lipid droplets compared with WT (Fig. $5 G$ and $H$ ). Other tissues were all normal.

Transmission electron microscope examination. The most striking observation in 4-wk-old mutant mice was the presence of abnormal mitochondria, which appeared swollen and rounded or elongated and even appeared multilamellar with loss of cristae and electron dense osmiophilic granules within the matrix compared with WT (Fig. 6A-C). Furthermore, stacks of paracrystalline inclusion bodies were observed in $10-30 \%$ of mitochondria in any given cell. This percentage was higher in the hepatocytes of 12-wk-old mutant mice (Fig. $6 D$ ). Hepatocytes from 30-wk-old mice showed increased damage to the mitochondria in the form of concentric cristae with amorphous matrix (Fig. 6E) and the formation of autophagosomes (Fig. 6F).

\section{DISCUSSION}

In this study, we describe the generation of mice with a mutation in Acad8, which had laboratory findings related to 

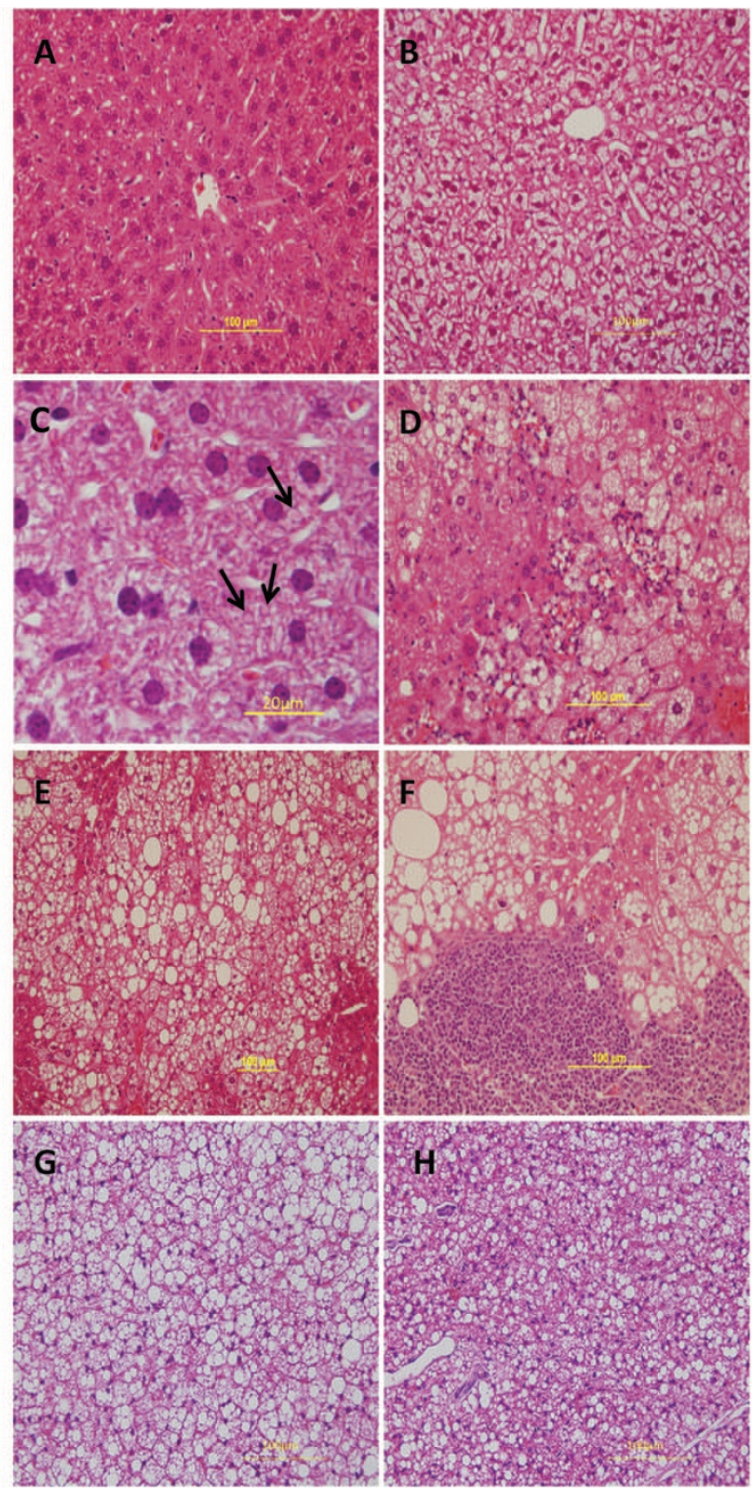

Figure 5. Histological analysis. (A) From 4-wk-old WT compared with the same age mutant mice $(B)$ indicated microvesicular steatosis in mutant mice with cytoplasmic red bodies (arrows) in mutant $(C)$. Twelve-week-old mutant mice $(D)$ shows progression from microvesicular to macrovesicular steatosis with mononuclear cell infiltration. Liver sections from 40 -wk-old mutant mice $(E$ and $F$ ) show macrovesicular steatosis with foci of mononuclear cells. BAT from 4-wk-old mice shows significantly smaller lipid droplets in sections from mutant mice $(G)$ compared with WT $(H)$. Scale bars: $A, B, D, E-H, 100$ $\mu \mathrm{m} ; \mathrm{C}, 20 \mu \mathrm{m}$.

IBD deficiency. We demonstrated that the mutation at position -17 of intron 1 was sufficient to activate an upstream aberrant 3 ' splice site. This resulted in alternative splicing, yielding an mRNA isoform containing the last 28 nucleotides of intron 1 with the eventual frame shift and the premature termination codon (16). In addition, we have sequenced Scad gene in which deficiency gives similar acylcarnitine profiles as IBD deficiency and found it to be normal (19). Furthermore, to rule out any possibility of second-site mutations could confound the phenotype analysis, we have further bred the affected mice with WT mice and carried out outcross breeding. All Acad8 mutant mice, now through the 12 th generation, continued to show $100 \%$ penetrance of the phenotypes.
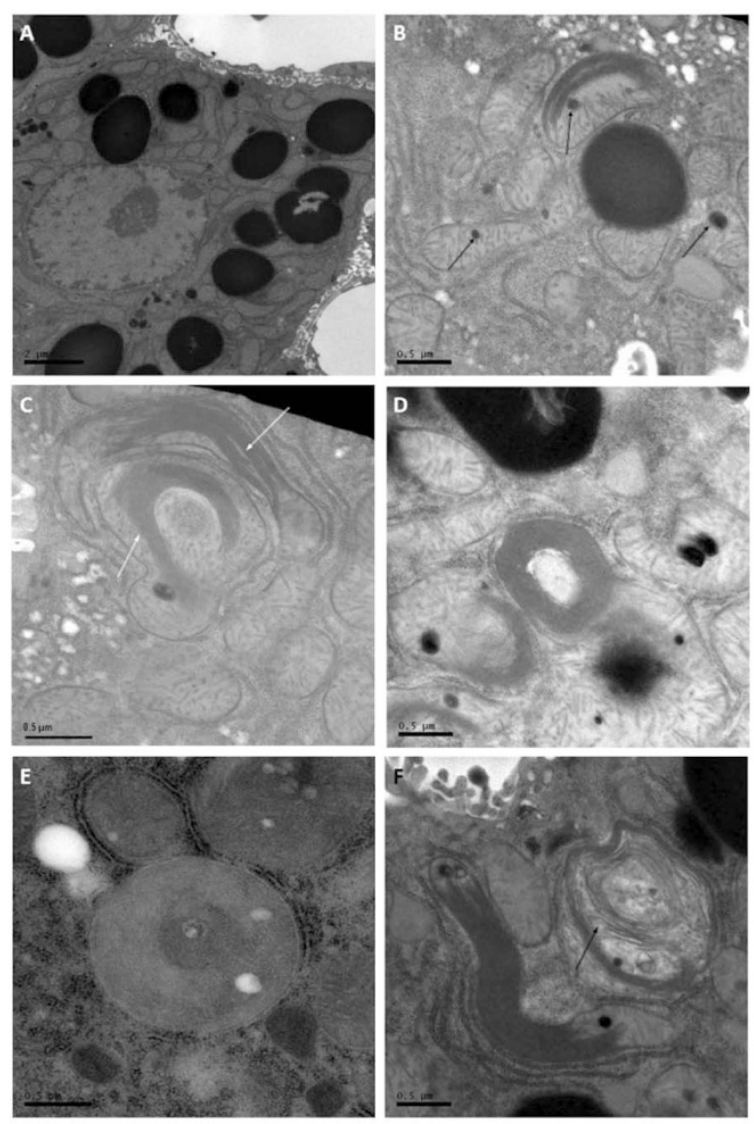

Figure 6. TEM study of the mutants' liver. Electron micrographs of 4-wk-old WT $(A)$ and mutant $(B$ and $C$ ) livers show the abnormal mitochondrial inclusions (arrows). Giant circular and elongated two mitochondria in $(C)$ with tubular- and shelf-like cristae closely packed. Twelve-week-old liver section $(D)$ shows concentric mitochondria cristae with amorphous matrix. Thirty-week-old liver $(E)$ shows the early formation of autophagosome, and 40-wk-old liver shows $(F)$ autophagosome (arrow) with long tubular mitochondria having shelf-like cristae. Scale bars: $A, 2 \mu \mathrm{m} ; B-F, 0.5 \mu \mathrm{m}$.

The published cases of human IBD deficiency are mild or asymptomatic $(6,8-11)$. Some patients have been shown to develop unexplained muscular hypotonia and cardiomyopathy. The heart and muscles of our mutant mice, however, did not show any histological or functional abnormalities. Our mice have defective metabolic rate, intolerance to cold, and abnormal mitochondria. These were not observed in patients because of either species differences or the lack of further follow-up. There is no reported liver involvement in patient with IBD deficiency, although liver biopsies were not done. By histological examination, we observed microvesicular steatosis in our mice, hepatocytes showed cytoplasmic red bodies suggested giant mitochondria (20). This was confirmed by transmission electron microscope (TEM) with the presence of osmiophilic granules and crystalline inclusions, which are known parameters for mitochondriopathy (21). At the present time, we do not know the exact mechanisms why these mice are showing global mitochondrial defects. We speculated that IBD deficiency resulted in the accumulation of the metabolite intermediate such as isobutyryl-CoA inside the mitochondria, which eventually create a status of mitochondriopathy with a subsequent defect of other mitochondrial 
functions as $\beta$-oxidation. As a result, there might be a compensatory lipogenesis resulting fatty liver. Moreover, overexpression of genes involved LXR pathway specially Srbplc and its downstream Acc suggested that malonyl-CoA production is increased with the subsequent increase in the lipogenesis (22). Even though there was no alterations in Cpt1, the rate-limiting step in $\beta$-oxidation, malonyl-CoA acts as a potent inhibiter, which intern decrease fatty acid $\beta$-oxidation (23) and these might be the cause of the development of fatty liver. Further studies will be needed to elucidate the mechanisms underlying the mitochondriopathy and hepatic insulin signaling and to see whether these mice represent a mouse model of IBD deficiency.

We observed that 4-wk-old mutant mice have a reduced capacity for thermoregulation (24). However, there was no difference in the expression of Ucp1 and its regulator Pgcla between the two groups after cold exposure. Defective $\beta$-oxidation might be the cause of cold intolerance. In contrast, mutant BAT fat droplets were smaller in size compared with WT, and older mice were more tolerant to cold. Mutant mice showed an alteration in the diurnal variation of RER, which might be a compensatory status. Administration of noradrenaline to mimic a hypermetabolic status only elevated the $\mathrm{VO}_{2}$ of the WT but not the mutant mice, which might be due to the defect in the $\beta$-oxidation pathway too.

In summary, we have identified a mouse strain with a novel IBD deficiency. With further investigations, these mice should provide us with a better understanding of the possible relation between IBD deficiency and fatty liver. As our mice demonstrated progressive fatty liver development and defective thermogenesis, we strongly recommend careful and long-term follow-up of human patients with IBD deficiency, because they might exhibit the same complications under stress conditions.

Acknowledgments. We thank Academia Sinica National Genotyping Center and Taiwan Mouse Clinic Core staff for their technical assistance. We also thank Dr. An-Li Huang for her technical assistance in performing transmission electron microscopy.

\section{REFERENCES}

1. Letto J, Brosnan ME, Brosnan JT 1986 Valine metabolism. Gluconeogenesis from 3-hydroxyisobutyrate. Biochem J 240:909-912

2. Hutson SM, Sweatt AJ, Lanoue KF 2005 Branched-chain [corrected] amino acid metabolism: implications for establishing safe intakes. J Nutr 135:1557S-1564S
3. Ramsay RR, Zammit VA 2004 Carnitine acyltransferases and their influence on CoA pools in health and disease. Mol Aspects Med 25:475-493

4. Ghisla S, Thorpe C 2004 Acyl-CoA dehydrogenases. A mechanistic overview. Eur J Biochem 271:494-508

5. Telford EA, Moynihan LM, Markham AF, Lench NJ 1999 Isolation and characterisation of a cDNA encoding the precursor for a novel member of the acyl-CoA dehydrogenase gene family. Biochim Biophys Acta 1446:371-376

6. Roe CR, Cederbaum SD, Roe DS, Mardach R, Galindo A, Sweetman L 1998 Isolated isobutyryl-CoA dehydrogenase deficiency: an unrecognized defect in human valine metabolism. Mol Genet Metab 65:264-271

7. Koeberl DD, Young SP, Gregersen NS, Vockley J, Smith WE, Benjamin DK Jr, An Y, Weavil SD, Chaing SH, Bali D, McDonald MT, Kishnani PS, Chen YT, Millington DS 2003 Rare disorders of metabolism with elevated butyryl- and isobutyryl-carnitine detected by tandem mass spectrometry newborn screening. Pediatr Res 54:219-223

8. Sass JO, Sander S, Zschocke J 2004 Isobutyryl-CoA dehydrogenase deficiency: isobutyrylglycinuria and ACAD8 gene mutations in two infants. J Inherit Metab Dis 27:741-745

9. Pedersen CB, Bischoff C, Christensen E, Simonsen H, Lund AM, Young SP, Koeberl DD, Millington DS, Roe CR, Roe DS, Wanders RJ, Ruiter JP, Keppen LD, Stein Q, Knudsen I, Gregersen N, Andresen BS 2006 Variations in IBD (ACAD8) in children with elevated C4-carnitine detected by tandem mass spectrometry newborn screening. Pediatr Res 60:315-320

10. Yoo EH, Cho HJ, Ki CS, Lee SY 2007 Isobutyryl-CoA dehydrogenase deficiency with a novel ACAD8 gene mutation detected by tandem mass spectrometry newborn screening. Clin Chem Lab Med 45:1495-1497

11. Oglesbee D, He M, Majumder N, Vockley J, Ahmad A, Angle B, Burton B, Charrow J, Ensenauer R, Ficicioglu CH, Keppen LD, Marsden D, Tortorelli S, Hahn SH, Matern D 2007 Development of a newborn screening follow-up algorithm for the diagnosis of isobutyryl-CoA dehydrogenase deficiency. Genet Med 9:108-116

12. Schuler AM, Wood PA 2002 Mouse models for disorders of mitochondrial fatty acid beta-oxidation. ILAR J 43:57-65

13. Wu JY, Kao HJ, Li SC, Stevens R, Hillman S, Millington D, Chen YT 2004 ENU mutagenesis identifies mice with mitochondrial branched-chain aminotransferase deficiency resembling human maple syrup urine disease. J Clin Invest 113:434-440

14. Kao HJ, Cheng CF, Chen YH, Hung SI, Huang CC, Millington D, Kikuchi T, Wu JY, Chen YT 2006 ENU mutagenesis identifies mice with cardiac fibrosis and hepatic steatosis caused by a mutation in the mitochondrial trifunctional protein beta-subunit. Hum Mol Genet 15:3569-3577

15. Weber JS, Salinger A, Justice MJ 2000 Optimal N-ethyl-N-nitrosourea (ENU) doses for inbred mouse strains. Genesis 26:230-233

16. Lin JC, Tarn WY 2005 Exon selection in alpha-tropomyosin mRNA is regulated by the antagonistic action of RBM4 and PTB. Mol Cell Biol 25:10111-10121

17. Golozoubova V, Cannon B, Nedergaard J 2006 UCP1 is essential for adaptive adrenergic nonshivering thermogenesis. Am J Physiol Endocrinol Metab 291:E350E357

18. Sanyal AJ, Campbell-Sargent C, Mirshahi F, Rizzo WB, Contos MJ, Sterling RK, Luketic VA, Shiffman ML, Clore JN 2001 Nonalcoholic steatohepatitis: association of insulin resistance and mitochondrial abnormalities. Gastroenterology 120:11831192

19. Bok LA, Vreken P, Wijburg FA, Wanders RJ, Gregersen N, Corydon MJ, Waterham HR, Duran M 2003 Short-chain Acyl-CoA dehydrogenase deficiency: studies in a large family adding to the complexity of the disorder. Pediatrics 112:1152-1155

20. Duong Van Huyen JP, Batisse D, Heudes D, Belair MF, Piketty C, Gonzalez-Canali G, Weiss L, Kazatchkine MD, Bruneval P 2006 Alteration of cytochrome oxidase subunit I labeling is associated with severe mitochondriopathy in NRTI-related hepatotoxicity in HIV patients. Mod Pathol 19:1277-1288

21. Ogawa K, Noguchi H, Tsuji M, Sasaki F 2003 Starvation induces the formation of giant mitochondria in gastric parietal cells of guinea pigs. J Electron Microsc (Tokyo) 52:217-225

22. Hue L, Taegtmeyer H 2009 The Randle cycle revisited: a new head for an old hat. Am J Physiol Endocrinol Metab 297:E578-E591

23. Gower BA, Nagy TR, Blaylock ML, Wang C, Nyman L 2002 Estradiol may limit lipid oxidation via Cpt 1 expression and hormonal mechanisms. Obes Res 10:167172

24. Trayhurn P, James WP 1978 Thermoregulation and non-shivering thermogenesis in the genetically obese (ob/ob) mouse. Pflugers Arch 373:189-193 